\title{
Treatment with Combined Resveratrol and Myoinositol Ameliorates Endocrine, Metabolic Alterations and Perceived Stress Response in Women with PCOS: A Double-Blind Randomized Clinical Trial
}

Sehresh Hassan

Khyber Medical University

Mohsin Shah ( $\square$ mohsin.ibms@kmu.edu.pk)

Khyber Medical University https://orcid.org/0000-0001-9017-9152

Muhammad Omar Malik

Khyber Medical University

Ehtesham Ehtesham

Khyber Medical University

Hamid Habib

Khyber Medical University

Bushra Rauf

Lady Reading Hospital

\section{Research Article}

Keywords: PCOS, Resveratrol, Myoinositol, Metformin, Pioglitazone, Adiponectin

Posted Date: February 24th, 2022

DOI: https://doi.org/10.21203/rs.3.rs-1341510/v1

License: (c) (i) This work is licensed under a Creative Commons Attribution 4.0 International License. Read Full License 


\section{Abstract}

Purpose: The present study aimed to assess whether treatment with combined resveratrol and myoinositol is more effective in ameliorating the altered parameters associated with PCOS when compared to the combined metformin and pioglitazone therapy.

Method: One hundred and ten obese, infertile PCOS women, aged 20-35 years were randomly assigned into two treatment arms. Participants in arm-1 $(n=55)$, received combination of metformin and pioglitazone (1000 mg and $30 \mathrm{mg}$, respectively), once daily, while those in arm-2 $(n=55)$ received combination of resveratrol and myoinositol (2000 mg and $2000 \mathrm{mg}$, respectively) once daily for 12 weeks. Evaluations performed at baseline were repeated after 3 months of therapy. The endocrine and metabolic derangements were assessed by measuring serum levels of testosterone, luteinizing hormone, follicle stimulating hormone, adiponectin and insulin using ELISA. Cohen's perceived stress scale (PSS) was employed as a subjective measure of stress.

Results: Pre-treatment PCOS women in both the arms (arm-1 and arm-2) had remarkably elevated serum testosterone and insulin concentrations, low serum adiponectin and high perceived stress response scores. The treatment reduced the altered endocrine indices in arm-2 (resveratrol and myoinositol) participants, manifested by statistically significant reduction in serum testosterone level $(p=0.001)$ and notably increased serum adiponectin level $(p=0.001)$. Interestingly, the hormonal profile, including serum LH and FSH levels also decreased $(p<0.001)$ along with a marked reduction in the ovarian volume $(p=0.001)$ in arm2 participants. There was a significant reduction in BMI $(p<0.001)$ and an improvement in waist-hip ratio $(p<0.001)$ in arm-2 participants compared to arm-1 group. The PSS scores of the arm-2 subjects improved significantly $(p<0.001)$ whereas, the Ferrimen-gallwey score was improved in both the arms (arm-1 and arm$2 ; p=0.010$ and 0.008 respectively) however, the change was highly significant in arm-2 (resveratrol and myoinositol). Interestingly, the menstrual regularity was $81.4 \%$ in arm-2 while $18.2 \%$ in arm- 1 . We conclude that the therapeutic intervention with combined resveratrol and myoinositol is more effective in ameliorating altered endocrine, metabolic indices and stress burden and could be of clinical importance in high risk group of obese, infertile married PCOS affected women.

Trial Registration: Trial registration: ClinicalTials.gov Trial No: NCT04867252. Registered 24 April, 2021, http://clinicaltrials.gov./ct2/show/NCT04867252

\section{Introduction}

Polycystic Ovary Syndrome (PCOS) is the prevalent heterogeneous endocrine-metabolic abnormality affecting $10-15 \%$ of women in their child bearing age $[1,2]$. It is a many-faceted health issue identified by chronic oligo or anovulation, hyperandrogenism and polycystic ovaries [3]. Apart from the clinical manifestations of PCOS, women with this syndrome are also accompanied by obesity, insulin resistance (IR), type II diabetes mellitus (T2DM), infertility, increased anxiety, fear of long term health complications along with depressive thoughts $[4,5]$. The etiology of this endocrine anbormailty is largely unknown but 
there are mounting evidences suggesting that PCOS is a multigenic health issue including both genetic and enviromental factors [6].

There is a broad range of remedial options with possible advantages available for PCOS manifestations, however several clinical studies are required to assess their safety and the clinical efficacy. Resveratrol is a natural polyphenol found in multiple fruits like skin of grapes, peanuts, red wine and many other medicines from the plants source [7]. This neutraceutical has antioxidant, antidiabetic and anti-inflammatory properties [8]. It inhibits alpha-amylase and alpha-glucosidase, leading to improved glycemic control; ultimately ameliorating diabetes and the complications associated with diabetes [9]. A wide range of favorable metabolic effects are attributed to resveratrol which includes enhancement of insulin sensitivity, balance in lipid profile and reduced adiposity [10]. Resveratrol improves glucose homeostasis and reverses insulin resistance through the activity of phosphodiesterases, kinases and SIR1 in multiple tissues and ultimately protects the pancreatic beta cells from degeneration [7]. Resveratrol has also shown to improve the number of degenerated and secondary follicles and decrease the number of graffian follicles in PCOS patients, this explains the significance of using resveratrol in maintaining folliculogenesis [11]. It has been reported to interact with multiple cell targets, but its major effects goes through the activation of silent information regulator1 (SIRT1), a protein that causes deacetylation of numerous other proteins involved in the homeostasis of glucose and energy [12]. The SIRT1 is mainly expressed in ovum and nuclei of human granulosa cells during different developmental stages of the follicles and exhibits repressive effects on inflammation in different cell types [13].

Myoinositol is a carbocyclic sugar integrated in the phospholipids of the cellular membrane, inositophosphoglycan and is considered to be an important part of structural lipids [14]. Foods rich in myoinositol and the associated lipids include corns, beans, nuts and fruits specially cantaloupe and oranges. $[15,16]$. The intracellular signal transduction of insulin involves myoinositol, when insulin binds to its receptors the transduction pathway is activated causing hydrolysis of phospholipids and production of intracellular messenger which causes oxidative metabolism of glucose [17]. Myoinositol acts as a predecessor to inositol triphosphate and regulates several hormones such as follicle-stimulating hormone (FSH) and insulin by acting as a second messenger [18]. Treating obese PCOS patients with inositols, without any lifestyle modifications is effective in reducing BMI [19]. The higher efficacy of myoinositol and the lower rate of side effects in comparison to the entrenched treatments like metformin and many other, it can be elucidated why myoinositol is considered when insulin lowering agents are suggested for PCOS women [20].

Metformin is a drug from the family of biguanides, used now a days as an antidiabetic drug for treating type II diabetes mellitus [21]. Metformin increases the cells sensitivity towards insulin by increasing the activity of tyrosine kinase in the insulin receptors that are present in adipocytes and muscles [22]. Pioglitazone is a member of thiazolidinedione (TZD) family with the hypoglycemic, antidiabetic action in treating insulin resistance in type II diabetes patients [23]. Because of its insulin sensitizing effects it can improve ovulation, the signs of excess androgen secretion, and may halt or can even retard the onset of diabetes [24]. 
In our previous studies, we evaluated the effect of metformin alone and metformin plus pioglitazone as standard treatment for PCOS women [25, 26]. Later on, we added acetyl-L-carnitine with the standard drug treatment and found significant amelioration of metabolic and endocrine response [27]. Here, we assessed the effectiveness of combined resveratrol and myoinositol on clinical, metabolic, endocrine alterations and stress burden in women with PCOS. The present trial was designed to address three aspects. Firstly, to compare the effectiveness of combined resveratrol and myoinositol with the combined metformin and pioglitazone therapy on serum testosterone, adiponectin and insulin levels in women with PCOS. Secondly, to evaluate the effects of 12 weeks intervention on hormonal profile in PCOS. Thirdly, to assess perceived stress response in PCOS women before and after 12 weeks of treatment.

\section{Subjects And Methods Study population}

The study population of this clinical trial comprised of 110, obese and infertile married PCOS women between the ages 20 and 35 years. The women, who fulfilled the diagnostic criteria for PCOS according to the Androgen Excess Society 2006 [28] were selected from outpatient clinic of gynecology Peshawar health center from May 2021 to August 2021. For the sample size calculation already available serum adiponectin values $11.3 \pm 4.8$ and $12.5 \pm 6.2 \mu \mathrm{g} / \mathrm{ml}$ [29] in PCOS affected women were used for comparing the values before the onset of the study and after 12 weeks by using $\mathrm{G}$ power software. With the a value of 0.05 and power of 0.95 , the sample size for the current trial was calculated as 88 .

\section{Inclusion criteria}

Inclusion of the participants in the study was based on the Androgen Excess Society (AES) 2006, according to this criteria a patient to be diagnosed with PCOS hyperandrogenism clinical or biological, must be accompanied with oligo or amenorrhea and/ or polycystic ovarian morphology on the ultrasonological examination. Participants with the menstrual cycle of greater than 35 days were classified as having oligomenorrhea while women with no menstruation for a period of 3 to 6 months or longer (after the pregnancy was ruled out) were classified as having amenorrhea. Clinical hyperandrogenism was interpreted by the presence of hirsutism, severe acne and/or hair loss. The polycystic ovarian morphology was elucidated on the pelvic ultrasound by either the follicular number per ovary of greater than 12 or more, measuring 2 to $9 \mathrm{~mm}$ in diameter or the ovarian volume exceeding $10 \mathrm{ml}$ in atleast one of the two ovaries.

\section{Exclusion criteria}

Participants on current medication known to influence the metabolic and endocrine profiles or treated with the hormone replacement therapy within the last three months were excluded from the present study. The women who were pregnant or on breastfeeding 12 weeks beofre the start of the study were excluded. Women having chronic diseases like diabetes mellitus, cushings disease, congenital adrenal hyperplasia or any other disease that mimicked PCOS like thyroid disorders or hyperprolactinemia were also excluded. The 
participants who failed to consume the treatment regimens for a duration of 3 months and those who were lost to the followup were also excluded.

\section{Study approval}

This trial received approval by the Advanced Study and Research Board of Khyber Medical University, ASRB approval No. DIR/KMU-AS\&RB/EC/001226 and the ethical approval by the institutional Ethical Review Committee, approval No. DIR/KMU-EB/EC/00076. After the approval all the study procedures were accomplished according to the institutional regulations and guidelines. The trial was registered at clinicalTrial.gov on April 24, 2021 with the trial registration No. NCT04867252. Web.

https://clinicaltrials.gov/ct2/show/NCT04867252.

\section{Study design}

The study protocol was based on the recommendations in the CONSORT statement. This study was a twoarm (1:1) randomized double blinded clinical trial. A total of 126 PCOS affected women were screened and among them 16 women, not fulfilling the inclusion criteria were excluded. The random assignment of the remaining 110 women by a trained researcher was effected in a double blind fashion; patients received either metformin combined with pioglitazone or resveratrol combined with myoinositol in consonance with the code supplied by the online computer generated randomizer.

\section{Randomization and Masking}

The researchers, participants and those who assessed the outcomes were blinded to the treatment and throughout the study could not recognize the actual treatment. The randomization code for creating the treatment assignment spread sheet for 1:1 allocation was obtained by using online Sealed Envelop software with a block of four and six randomization technique. In order to eradicate any possible bias, the pre-treatment and the post-treatment evaluations of all the participating women were performed by the same investigator.

\section{Patients allocation and treatments}

All the PCOS affected women before the onset of any trial related intervention provided their informed written consent. The participants were randomized and assigned (1:1) into two treatment arms (1 and 2) for a period of 12 weeks. Each arm comprised of 55 women after being randomized and received the following treatments: 55 women in the conventional arm-1 recieved the combination of metformin and pioglitazone (1000 and $30 \mathrm{mg}$, orally,once daily); 55 women in the interventional arm-2 received the combination of resveratrol and myoinositol (2000 and $2000 \mathrm{mg}$, orally, once daily). The patients were properly guided on how to take the treatment regimens, to come for the follow-up visit and a reminder prior to the clinic visit was sent to participants. The participating women were also instructed to avoid any kind of drugs with possible metabolic and endocrine effects throughout the study period.

\section{Treatment discontinuation}


The treatment discontinuation was advised to the participants in case they got pregnant, had any serious side effects or being diagnosed with disease included in the exclusion criteria. However, no adverse events were reported in both the treatment arms during the study period.

\section{Clinical measures}

The participant's demographic data and anthropometric measurements were recorded using standard techniques. BMI was calculated by using weight in kilograms/height in meter ${ }^{2}$. Participant's body circumferences were measured with the stretch-resistant tape. The waist circumference was measured at a midpoint between the least palpable lower rib and the top of iliac crest. The hip circumference was measured around the widest portion of the buttocks with the tape parallel to the floor. The blood pressures of all the participating women were recorded using Omron Gold blood pressure monitor. Hyperandrogenemia was assessed clinically by the presence of excess, thick body hair and facial acne in the PCOS women. To grade hirsutism Ferrimen Gallwey scale [30] was used including the nine different areas of the body including upper lip, chin, chest, upper abdomen, lower abdomen, arms, thighs, upper back and lower back with the score ranging from 1-4 for each area. Ovarian morphology was assessed by transabdominal ultrasound after an hour of 24 ounces of water consumption. The ultrasound transducer was gently pressed against the abdomen moving it back and forth that displayed the images on the computer screen and the ovarian volume on both the sides was estimated using the simplified formula: $0.5 \times$ length $\times$ width $\times$ thickness.

\section{Venous blood sample collection:}

For the analysis of serum testosterone, adiponectin, insulin and LH, FSH levels venous blood samples from the antecubital vein were collected from the study participants. Serum was extracted from the blood by centrifugation at $3000 \mathrm{rpm}$ for a total of 10 minutes. The collected transparent supernatant was transferred to the Eppendorf tubes and was stored at $-80^{\circ} \mathrm{C}$ until analysis.

\section{Hormone assays:}

Commercially available immunosorbent assay kits were used for the analysis of adiponectin, insulin and testosterone. Adiponectin ELISA kit purchased from Elabscience, US (Cat. No. E-EL-H5811), Insulin from Perkin Elmer, USA (Cat.No.10801) \& Testosterone from Calbiotech, USA (Cat No. TE373S) and run according to the instructions provided by the manufacturers. The results were interpreted by two independent observers aside from the primary investigators who were blind to the status of the subjects.

\section{Psychological Assessment:}

For stress and psychological evaluation of the study participants we used Sheldon Cohen perceived stress scale (PSS-14). This 14 item instrument was designed to find out how unforeseeable, uncontrollable and overburdened the study participants found their lives. The questions asked from the women were related to the thoughts and feelings during the last month and the responses varied from 0 to 4 , i.e. $0=$ never, $1=$ almost never, $2=$ sometimes, 3 =fairly often and 4=very often respectively. To calculate the total stress score of the individual participant, the response of the participants to the seven positive questions, i.e. 4, 5, 6, 9, 10, 11 and 13 were reversed, i.e. $0=4,1=3,2=2,3=1 \& 4=0$. Finally the response to all the 14 questions were 
summed up that provided the total stress score of the participant. Score from 0 to 4 (never, almost never, sometimes, fairly often and very often respectively). The total PSS score for an individual ranged from 0 to 56 . Based on the total scores, participants were categorized as women with low (0-13), moderate (14-26) and high (27-56) perceived stress.

\section{Statistical analysis}

Analysis was carried out using SPSS-20 statistical software. The demographic data of the patients was evaluated at baseline as descriptive statistics and was presented as mean \pm standard deviation. The normally distributed variables were compared between the two treatment arms by using independentsample t-test. Paired t-test was applied to evaluate the changes between the measurements at baseline and after 12 weeks of intervention. All variables were normally distributed except for insulin and testosterone, Chi-square test was used to compare the categorical variables in the study and their response to the treatment regimens in the two treatment arms. $\mathrm{P}<0.05$ was considered significant.

\section{Results}

\section{PCOS women were randomly assigned to the different treatment arms (arm-1 and arm-2)}

A total of 126 PCOS women were screened for the eligibility. Out of them 16 women for various reasons were considered ineligible and the remaining 110 eligible women were allocated randomly for the 12 weeks intervention into the two treatment groups. The flow sheet of the participant's recruitment and randomization is shown in Figure 1. The modified intention to treat analysis included 55 patients in each intervention group. The arm-1 participants received conventional drug treatment of metformin and pioglitazone (1000 mg and $30 \mathrm{mg}$ once daily, respectively) and the interventional arm-2 participants received resveratrol and myoinositol (2000 mg and $2000 \mathrm{mg}$ once daily, respectively). A total of 22 subjects were lost to follow up ( 11 from arm-1 and 11 from arm-2). The final cohort available for study analysis included 88 participants ( 44 in arm-1 and 44 in arm-2). Treatment discontinuation was advised to the participants who got pregnant or had adverse reaction. However no major side effects were reported in this three month intervention from either group.

\section{PCOS women treated with resveratrol and myoinositol manifest remarkable amelioration of anthropometric and hemodynamic profiles}

Both the treatment regimens had influenced the anthropometric profile in PCOS patients however, the combination of resveratrol and myoinositol (arm-2) significantly reduced the weight and the BMI (both $\mathrm{p}<0.001)$ as compared to the metformin and pioglitazone (arm-1) where no statistical significance was achieved. Likewise, there was no decrease in waist and hip circumference and WHR in arm-1, however, there was a significant decrease in both waist-hip circumference and WHR in arm-2 (both $p<0.001$ ) as shown in Table 1. The mean systolic and diastolic BP in the two treatment arms at baseline were in the normal range. After treatment for 12 weeks, the mean systolic $(p=0.002)$ and diastolic BP $(p=0.001)$ decreased significantly in the arm- 2 as compared to arm- $1(p=0.247$ and $p=0.453$, respectively) as shown in Table 1 . 
PCOS affected women treated with resveratrol and myoinositol showed significant improvement in the endocrine parameters

Hormonal parameters between the two arms are shown in Table 1. As compared to the baseline, the significant decrease was observed in the levels of serum LH [14.753 \pm 6.0492 to $9.045 \pm 3.239 ; p<0.001]$; serum FSH [8.165 \pm 4.205 to $6.116 \pm 2.3005 ; \mathrm{p}<0.001]$ and serum testosterone $[0.65(0.55-0.77)$ to $0.37(0.27-0.51) ; p<0.001]$ in the interventional arm-2 after 12 weeks therapy. The values for testosterone were log transformed for analysis, values presented as geometric mean (confidence interval). Conversely, there were no significant changes in the hormonal measures in the conventional arm-1 treated PCOS women.

\section{PCOS affected women treated with resveratrol and myoinositol exhibited significant improvement in the metabolic parameters}

Interestingly here in this study we observed a remarkable increment in the serum adiponectin level in arm-2 when compared to the baseline concentration [7.114 \pm 3.400 to $8.870 \pm 0.43027 ; p<0.001]$ as shown in Figure 2. Whereas, no significant increment in the adiponectin concentration was observed in arm-1 [5.908 \pm 3.0918 to $4.955 \pm 4.984 ; p=0.15$ ] showing the enhanced efficacy of combined resveratrol and myoinositol in improving the adiponectin level in PCOS women. Adiponectin, an adipocytes derived hormone is an important homeostatic factor regulating blood glucose levels, insulin sensitivity and lipid metabolism [31]. PCOS affected women have low serum adiponectin level which is largely attributed to obesity and insulin resistance common in these women [32].

Effects of the two different therapies on serum insulin level are shown in Figure 3. A significant decrease in the level of insulin was observed in women with PCOS in the arm-2 group who were treated with the combination of resveratrol and myoinositol [40.43(30.46-53.66) to 25.57(18.92-34.56); $p=0.002]$ while no statistically significant difference was observed in the arm-1 group [21.53(16.95-27.35) to 23.17(18.5528.94; $p=0.56]$.

PCOS affected women treated with resveratrol and myoinositol demonstrate substantial depletion in ovarian volumes and menstrual irregularity: The mean ovarian volume of both the ovaries prior to the treatment was extremely high $(>10 \mathrm{ml})$ in the study population of both the treatment arms. After 12 weeks of resveratrol and myoinositol therapy, both the right and left sided ovarian volumes reduced significantly (Figure 4A and B). In the arm-1, there was a significant decrease in left ovarian volume only (Figure 4A).

All participants in both arms had irregular menstrual cycles before the onset of treatment. After 12 weeks of intervention $84.1 \%$ women in the arm 2 had regular cycles, and only $23.4 \%$ participants in arm 1 got menstrual regularity (Table 2).

PCOS affected women treated with resveratrol and myoinositol demonstrate considerable lessening of the dermatological issues 
The total Ferrimen-Galleway (FG) score evaluating hirsutism for the 9 different areas of the body is shown in Table 1. The total FG scores before treatment was very high in the study population of both the treatment arms. Following 12 weeks of therapy, there was a significant diminution in the FG scores in both the treatment arms. However, the difference was highly significant in the resveratrol and myoinositol arm-2 $(p=0.008)$ in comparison to the metformin and pioglitazone arm-1 $(p=0.010)$ as summarized in Table. 1.

The comparison of pre and post-treatment acne score between the conventional and interventional arms participants is shown in Table 2. The participating PCOS women in both the arms were divided into two data sets, according to those who have got acne and those without acne. In the conventional arm- 1 and the interventional arm-2, $57.4 \%$ and $31.8 \%$ had acne respectively, while $42.5 \%$ and $68.2 \%$ were without acne respectively. At baseline there was no statistically significant difference between the two arms $(p=0.10$, chisquare test). After follow up for 12 weeks treatment with resveratrol and myoinositol in arm-2 participants showed significant improvement with $90.9 \%$ clarity from acne, where as $46.8 \%$ in the metformin and pioglitazone arm-1. The difference between the two arms after 12 weeks follow up was statistically significant (both $p=0.000$, chi-square test).

\section{PCOS affected women treated with resveratrol and myoinositol showed immense amelioration of the stress load}

The perceived stress score (PSS) at baseline were high in both the treatment arms intimating that PCOS affected women in comparison to the healthy women experiences excessive perceived stress (Figure. 5), indicating more negative stress in these women. After 12 weeks therapy with combined resveratrol and myoinositol (arm-2), the PSS scores were reduced significantly $(p<0.001)$, but no significant difference was observed in the metformin and pioglitazone $(\mathrm{arm}-1)$ treated group $(\mathrm{p}=0.71)$.

\section{Discussion}

Polycystic ovary syndrome is known to be a more complexed rather than a simple reproductive disorder and involves a vicious cycle of endocrine/reproductive and metabolic disarray. The current study reports the deranged endocrine, metabolic parameters and stress burden in women affected with PCOS and reveals that the treatment intervention with combined resveratrol and myoinositol for a period of twelve weeks significantly alleviated different alterations associated with this disorder. Women with PCOS suffer from multifarious derangements in endocrine, metabolic indices and present with extreme stress response and treatment with the standard drugs including different insulin sensitizers alone or along with the contraceptive pills may relieve the signs and symptoms associated with syndrome; however there is no efficient and safe medicinal intervention yet to alleviate the signs and symptoms associated with this multifaceted disorder. Here, the current clinical trial supports that the improvement in these alterations in response to combined resveratrol and myoinositol is comparable to or rather much more pronounced than that of the observed combined effect of metformin and pioglitazone. This combined regimen was very well tolerated with none of the participants requiring discontinuation due to any intolerable side effects and the treatment resulted in the improvement of the anthropometric measures including a significant decrement in weight, BMI, waist-hip circumference and waist-to-hip ratio (Table.1). We also reported that the use of 
aforementioned remedy ameliorated the endocrine, metabolic alterations and relieved stress load along with the resumption of regular menstrual cycles in women with oligo/amenorrhea (Table. 2).

It has been previously reported that approximately $44 \%$ of PCOS women are obese and an adipocytederived hormone, adiponectin might be involved for the endocrine and metabolic derangements [33, 34]. Obesity increases the risk of co-morbidities associated with PCOS, such as impaired glucose tolerance and type II diabetes mellitus, hyperlipidemia and hypoadiponectinemia. Orio et al., evaluated the levels of serum adiponectin in a cohort of obese PCOS and revealed that adiponectin level was significantly low in these women [35]. Several other studies have reported that adiponectin level is significantly low in PCOS women, making them more susceptible to the likelihood of developing metabolic syndrome and future myocardial infarction [36]. Another similar study by Panidis et al., found that PCOS women with the BMI greater than 25 $\mathrm{kg} / \mathrm{m}^{2}$ had low serum adiponectin level when compared to the women with BMI less than $25 \mathrm{~kg} / \mathrm{m}^{2}$ [37]. Currently, to the best of our knowledge there is no available literature on the effects of resveratrol alteration of serum adiponectin level in PCOS women. However, Mousavi et al,. analyzed the effects of resveratrol on body weight, body mass index and waist circumference in obese adults and revealed a notable reduction in the weight parameters [38]. Le Donne et al., reported a remarkable depletion in the body weight, BMI, waist and hip circumferences while treating obese PCOS women with 4 grams per day of myoinositol for three months [39]. Here, we included only obese PCOS women with the BMl greater than $30 \mathrm{~kg} / \mathrm{m}^{2}$ and their serum adiponectin level were found significantly low. The current finding is in agreement with those of Tschritter et al., [40] and Pangaribuan et al., [41] who reported that adiponectin can be suppressed by increased body fat. Furthermore, the current study shows that combined treatment with resveratrol and myoinositol significantly augmented serum adiponectin level along with the remarkable reduction in $\mathrm{BMI}$, waist and hip circumference and waist to hip ratio when compared to metformin and pioglitazone arm-1 participants (Table. 1 and Figure. 2).

Subsequent studies have reported that PCOS, in addition to the reproductive dysfunction is also associated with the broad range of metabolic and endocrine derangements [42-44]. Several researchers have delineated the hormonal disorders such as raised circulating levels of androgens and hyperinsulinemia as well as the increased secretion of gonadotropins, as the key aspect of PCOS etiology [45, 46]. Ovarian hyperandrogenism in PCOS is mainly attributed to an inherent steroidogenic defect of theca cells [47]. The first clinical trial by Banaszewska et al., evaluated the effects of resveratrol $1500 \mathrm{mg}$ per day on PCOS patients and reported a significant decline in serum testosterone and DHEAS levels through the melioration of insulin resistance [48]. Interestingly, Bahramrezaie et al., reported a significant reduction in serum LH and testosterone in PCOS affected women with the use of resveratrol $800 \mathrm{mg}$ per day for a 40 days period [49]. Pizzo et al., evaluated the effects of myoinositol on endocrinological PCOS aspects and reported a significant depreciation of LH, LH/FSH ratio and serum testosterone level [50]. In a study by Genazzani et al,. plasma LH, testosterone, insulin levels and LH/FSH ratio significantly reduced after 12 weeks of myoinositol administration [51]. Effects of resveratrol and myoinositol on hormonal profile of PCOS have been checked separately in different studies. However, to the best of our knowledge no study has reported the combined effect of resveratrol and myo-inositol hitherto. In the present study where both the drugs were combined, we found significant amelioration of the metabolic, endocrine response as previously reported 
studies, however, our results were astonishing in the sense that the combination therapy significantly enhanced most of the clinical and biochemical parameters compared to the standard drug regimen. Moreover the present study also delineated the significant lessening in the dermatological manifestations associated with the hormonal malfunctions, including hirsutism (FG scores $<0.001$ ) and acne scores (Table. 2).

The massive disappointment for women with PCOS is their inability to conceive as it is considered to be the most common cause of anovulatory infertility [52]. Oligomenorrhoea/ anovulation is a major clinical concern and is present in $70-80 \%$ of women with PCOS [53]. Several studies suggest that the hormonal imbalance associated with PCOS might be the cause of subfertility issue [54]. The effect of resveratrol has been insufficiently studied on the abnormal menstrual cycles in PCOS. Benrik et al., while evaluating the effects of resveratrol in rats with androgen-induced PCOS, reported significant enhancement of menstrual cycles [55]. Mansour et al., recently reported improved menstrual irregularities with the use of natural resveratrol 1gram, once daily for the three months duration [56]. Papaleo et al., evaluated the effects of myoinositol 2 grams per day on the menstrual irregularity in PCOS and demonstrated restoration of spontaneous ovulation and menstrual cycles [57]. The present results are in line with other studies, reporting a significant reduction in the volumes of both the ovaries and normalization of menstrual cycles. Interestingly, in our study regular menstruation was restored in $84.1 \%$ (Table.2) in combined resveratrol and myoinositol group along with the substantial depletion in the ovarian volumes (Figure. 4).

Insulin resistance and the associated compensatory hyperinsulinemia are frequently observed dysfunctions in PCOS [58]. Women with PCOS are insulin resistant and are at higher risk of glucose intolerance which may lead to crucial metabolic consequences with an increased possibility of developing type II diabetes mellitus [59]. Ortega et al., suggested that resveratrol has been observed to have antidiabetic effect [60]. Hoseini et al., investigated the effects of $500 \mathrm{mg}$ per day resveratrol for 4 weeks on the metabolic status of the type II diabetic patients and reported significantly reduced levels of insulin and HOMA-IR score [61]. The effect of resveratrol on the regularization of insulin sensitivity has been reported previously $[62,63]$. Talbott et al., proposed periodic screening of PCOS women for the timely detection of diabetes [64]. Costantino et al., performed a double-blind trial in which 23 insulin resistant PCOS women were treated with 4 gram once daily of myoinositol for 12 weeks that resulted in a significant reduction in the circulating insulin levels and improved glucose tolerance [65]. A similar study by Fruzzetti et al., reported that myoinositol is effective in diminishing serum insulin levels [66]. Our study is similar to the above stated studies where we observed a significant reduction in serum insulin levels in both the arms but reduction was statistically significant in arm-2 participants (Figure. 3).

Increased rates of stress, anxiety and depression in PCOS affected women are irrefutable throughout the literature [67-69]. Several studies suggest that PCOS women exhibit up to four fold increased risk of depressive symptoms and six fold risk of anxiety symptoms compared with normal women [68, 70, 71]. The observations made in a recent study by Tianyao et al., indicated that resveratrol administration alleviated the anxiety and depression like symptoms in ovariectomized rats [72]. The potency of resveratrol in treating depression and anxiety was confirmed by several other studies $[73,74]$. Here, in this study we used Cohen's perceived stress scale to evaluate perceived stress response in PCOS women and revealed significant 
reduction in PSS scores in participants given combined resveratrol and myoinositol for 12 weeks (Figure. $5)$.

This study, to the best of our knowledge is the first of its kind, assessing the efficacy of 12 weeks supplementation with combined resveratrol and myoinositol while addressing multiple outcome variables in women with PCOS. This randomized, double-blinded trial reports that the extent of betterment in the metabolic, endocrine alterations and stress burden observed in the PCOS affected women in response to combined resveratrol and myoinositol supplementation is comparable to or more pronounced than that observed in response to the combined use of metformin and pioglitazone.

The most important limitation to this study was that there were no separate groups to evaluate the efficacy of resveratrol and myoinositol alone on the altered parameters in PCOS affected women.

In order to confirm our data and to make it more obvious that the above-stated combination regimen can be recommended in the clinical situation, further randomized clinical trials with extended duration are warranted to be carried out in women with PCOS. The findings of this study could have public health importance and clinical implications for high risk PCOS women.

\section{Conclusion}

The data reported for the combined resveratrol and myoinositol supplementation (2 grams each for 12 weeks) is encouraging and offers therapeutic option to the first line treatment in PCOS women. The study conclusively proves that this combination therapy has a definitive role in relieving the clinical, endocrine, metabolic malfunctions and stress burden in PCOS. Therefore, further studies are needed to confirm these results; it could be considered potential therapeutic alternative to traditional drugs, without noticeable effects.

\section{Declarations}

Compliance with ethics guidelines: All procedures involving human participants performed in this study were in accordance with the ethical standards of Khyber Medical University's ethical committee and with the 1964 Helsinki declaration and its later amendments or comparable ethical standards. The study was approved by the institution board of study and informed consent was obtained from each participants included in the study.

Acknowledgment: We thank the study subjects for participating in this clinical trial.

Disclosure: The authors Sehresh Hassan, Mohsin Shah, Ehtesham, Hamid Habib, Bushra Rauf and Muhammad Omar Malik, have nothing to disclose. The authors alone are responsible for the content and writing of the paper. Natural resveratrol (Batch No. RY20201015) and myoinositol (Batch No. RY20201004) was purchased from a pharmaceutical company in China, Baoji Runyo Bio-technology Ltd. Metformin and Piogiltazone were purchased from Getz pharmaceutical company. The pharmaceutical company had no other role in the current study. 
Funding: No specific fund received.

Author's contributions: Sehresh Hassan was involved in the execution of project writing of the manuscript. Mohsin Shah supervised, designed, executed the study and wrote the manuscript. Bushra Rauf cosupervised the study and provided clinical expertise. Muhammad Omar Malik helped in organization of data and did the statistical analysis. Ehtesham and Hamid Habib did critical editing of the manuscript. All named authors approved the final version of the manuscript. "All named authors meet the International Committee of Medical Journal Editors (ICMJE) criteria for authorship for this article, take responsibility for the integrity of the work as a whole, and have given their approval for this version to be published."

Data availability: Available from the corresponding author on reasonable request.

\section{References}

1. A.L. Rocha et al., Recent advances in the understanding and management of polycystic ovary syndrome. 2019. 8

2. G. Monastra et al., Combining treatment with myo-inositol and D-chiro-inositol (40:1) is effective in restoring ovary function and metabolic balance in PCOS patients. Gynecol. Endocrinol. 33(1), 1-9 (2017)

3. S.M. Sirmans, K.A. Pate, Epidemiology, diagnosis, and management of polycystic ovary syndrome. Clin. Epidemiol. 6, 1-13 (2013)

4. D.A. Ehrmann, Polycystic ovary syndrome. N Engl. J. Med. 352(12), 1223-1236 (2005)

5. M. Bani Mohammad, A. Majdi Seghinsara, Polycystic Ovary Syndrome (PCOS), Diagnostic Criteria, and AMH. Asian Pac. J. Cancer Prev. 18(1), 17-21 (2017)

6. E.K. Barthelmess, R.K. Naz, Polycystic ovary syndrome: current status and future perspective. Front. Biosci. (Elite Ed) 6, 104-119 (2014)

7. B. Salehi, A.P. Mishra, Resveratrol: A Double-Edged Sword in Health Benefits. 2018. 6(3)

8. S. Guo et al., Resveratrol attenuates high glucose-induced oxidative stress and cardiomyocyte apoptosis through AMPK. Mol. Cell. Endocrinol. 412, 85-94 (2015)

9. O.R. Oyenihi, A.B. Oyenihi, Antidiabetic Effects of Resveratrol: The Way Forward in Its Clinical Utility. 2016. 2016: p. 9737483

10. K. Szkudelska, T. Szkudelski, Resveratrol, obesity and diabetes. Eur. J. Pharmacol. 635(1), 1-8 (2010)

11. S. Furat Rencber et al., Effect of resveratrol and metformin on ovarian reserve and ultrastructure in PCOS: an experimental study. J. Ovarian Res. 11(1), 55 (2018)

12. A. Ochiai, K. Kuroda, Preconception resveratrol intake against infertility (Friend or foe? Reproductive Medicine and Biology, 2019)

13. C. Tatone et al., Sirtuin Functions in Female Fertility: Possible Role in Oxidative Stress and Aging. Oxidative Medicine and Cellular Longevity, 2015. 2015: p. 659687 
14. P.-A. Regidor, A.E. Schindler, Myoinositol as a Safe and Alternative Approach in the Treatment of Infertile PCOS Women: A German Observational Study. International Journal of Endocrinology, 2016. 2016: p. 9537632

15. M. Bizzarri et al., Pharmacodynamics and pharmacokinetics of inositol(s) in health and disease. Expert Opin. Drug Metab. Toxicol. 12(10), 1181-1196 (2016)

16. R.S. Clements Jr., B. Darnell, Myo-inositol content of common foods: development of a high-myoinositol diet. Am. J. Clin. Nutr. 33(9), 1954-1967 (1980)

17. S. Suzuki et al., Urinary chiro-inositol excretion is an index marker of insulin sensitivity in Japanese type II diabetes. Diabetes Care 17(12), 1465-1468 (1994)

18. G. Di Paolo, P. De Camilli, Phosphoinositides in cell regulation and membrane dynamics. Nature 443(7112), 651-657 (2006)

19. F. Saleem, S.W. Rizvi, New Therapeutic Approaches in Obesity and Metabolic Syndrome Associated with Polycystic Ovary Syndrome. Cureus 9(11), e1844 (2017)

20. M.F. Gangale et al., Long-term metformin treatment is able to reduce the prevalence of metabolic syndrome and its hepatic involvement in young hyperinsulinaemic overweight patients with polycystic ovarian syndrome. Clin. Endocrinol. (Oxf) 75(4), 520-527 (2011)

21. Reis, F.M.

22. L.S. Hermann, Metformin: a review of its pharmacological properties and therapeutic use. Diabete Metab. 5(3), 233-245 (1979)

23. U. Smith, Pioglitazone: mechanism of action. Int J Clin Pract Suppl, 2001(121): p. 13-8

24. Y. Xu, Y. Wu, Q. Huang, Comparison of the effect between pioglitazone and metformin in treating patients with PCOS:a meta-analysis. 2017. 296(4): p. 661-677

25. D.E. Ali et al., Treatment with Metformin and Combination of Metformin Plus Pioglitazone on Serum Levels of IL- 6 and IL-8 in Polycystic Ovary Syndrome: A Randomized Clinical Trial. Horm. Metab. Res. 51(11), 714-722 (2019)

26. M. Zahra et al., Effects of Metformin on Endocrine and Metabolic Parameters in Patients with Polycystic Ovary Syndrome. Horm. Metab. Res. 49(2), 103-108 (2017)

27. S. Tauqir et al., Acetyl-L-Carnitine Ameliorates Metabolic and Endocrine Alterations in Women with PCOS: A Double-Blind Randomized Clinical Trial. 2021. 38(7): p. 3842-3856

28. R. Azziz et al., The Androgen Excess and PCOS Society criteria for the polycystic ovary syndrome: the complete task force report. Fertil. Steril. 91(2), 456-488 (2009)

29. M. Luque-Ramírez, F. Alvarez-Blasco, H.F. Escobar-Morreale, Antiandrogenic contraceptives increase serum adiponectin in obese polycystic ovary syndrome patients. Obes. (Silver Spring) $17(1), 3-9$ (2009)

30. R. Aswini, S. Jayapalan, Modified Ferriman-Gallwey Score in Hirsutism and its Association with Metabolic Syndrome. Int. J. Trichology 9(1), 7-13 (2017)

31. T.M.D. Nguyen, Adiponectin: Role in Physiology and Pathophysiology. Int. J. Prev. Med. 11, 136 (2020) 
32. S.S. Mirza et al., Association between circulating adiponectin levels and polycystic ovarian syndrome. J. Ovarian Res. 7, 18 (2014)

33. E. Carmina, R.A. Lobo, Polycystic ovary syndrome (PCOS): arguably the most common endocrinopathy is associated with significant morbidity in women. J. Clin. Endocrinol. Metab. 84(6), 1897-1899 (1999)

34. W.S. Yang et al., Weight reduction increases plasma levels of an adipose-derived anti-inflammatory protein, adiponectin. J. Clin. Endocrinol. Metab. 86(8), 3815-3819 (2001)

35. F. Orio Jr. et al., Adiponectin Levels in Women with Polycystic Ovary Syndrome. J. Clin. Endocrinol. Metabolism 88(6), 2619-2623 (2003)

36. D. Glintborg et al., Evaluation of metabolic risk markers in polycystic ovary syndrome (PCOS). Adiponectin, ghrelin, leptin and body composition in hirsute PCOS patients and controls. Eur. J. Endocrinol. 155(2), 337-345 (2006)

37. D. Panidis et al., Serum adiponectin levels in women with polycystic ovary syndrome. Hum. Reprod. 18(9), 1790-1796 (2003)

38. S.M. Mousavi, A. Milajerdi, Resveratrol supplementation significantly influences obesity measures: a systematic review and dose-response meta-analysis of randomized controlled trials. 2019. 20(3): p. $487-498$

39. M. Le Donne et al., Effects of three treatment modalities (diet, myoinositol or myoinositol associated with D-chiro-inositol) on clinical and body composition outcomes in women with polycystic ovary syndrome. Eur. Rev. Med. Pharmacol. Sci. 23(5), 2293-2301 (2019)

40. 0 . Tschritter et al., Plasma adiponectin concentrations predict insulin sensitivity of both glucose and lipid metabolism. Diabetes 52(2), 239-243 (2003)

41. B. Pangaribuan et al., Serum adiponectin and resistin in relation to insulin resistance and markers of hyperandrogenism in lean and obese women with polycystic ovary syndrome. Ther. Adv. Endocrinol. Metab. 2(6), 235-245 (2011)

42. L.C. Morin-Papunen et al., Insulin sensitivity, insulin secretion, and metabolic and hormonal parameters in healthy women and women with polycystic ovarian syndrome. Hum. Reprod. 15(6), 1266-1274 (2000)

43. E. Diamanti-Kandarakis et al., Inflammatory and endothelial markers in women with polycystic ovary syndrome. Eur. J. Clin. Invest. 36(10), 691-697 (2006)

44. A. Dunaif, Insulin resistance and the polycystic ovary syndrome: mechanism and implications for pathogenesis. Endocr. Rev. 18(6), 774-800 (1997)

45. P. Moghetti, Insulin Resistance and Polycystic Ovary Syndrome. Curr. Pharm. Des. 22(36), 5526-5534 (2016)

46. S. Arentz et al., Herbal medicine for the management of polycystic ovary syndrome (PCOS) and associated oligo/amenorrhoea and hyperandrogenism; a review of the laboratory evidence for effects with corroborative clinical findings. BMC Complement. Altern. Med. 14, 511 (2014)

47. E. Diamanti-Kandarakis, Polycystic ovarian syndrome: pathophysiology, molecular aspects and clinical implications. Expert Rev. Mol. Med. 10, e3 (2008) 
48. B. Banaszewska et al., Effects of Resveratrol on Polycystic Ovary Syndrome: A Double-blind, Randomized, Placebo-controlled Trial. J. Clin. Endocrinol. Metab. 101(11), 4322-4328 (2016)

49. M. Bahramrezaie, F. Amidi, Effects of resveratrol on VEGF \& HIF1 genes expression in granulosa cells in the angiogenesis pathway and laboratory parameters of polycystic ovary syndrome: a triple-blind randomized clinical trial. 2019. 36(8): p. 1701-1712

50. A. Pizzo, A.S. Laganà, L. Barbaro, Comparison between effects of myo-inositol and D-chiro-inositol on ovarian function and metabolic factors in women with PCOS. Gynecol. Endocrinol. 30(3), 205-208 (2014)

51. A.D. Genazzani et al., Myo-inositol administration positively affects hyperinsulinemia and hormonal parameters in overweight patients with polycystic ovary syndrome. Gynecol. Endocrinol. 24(3), 139144 (2008)

52. R. Hart, PCOS and infertility. Panminerva Med. 50(4), 305-314 (2008)

53. R. Azziz et al., Positions statement: criteria for defining polycystic ovary syndrome as a predominantly hyperandrogenic syndrome: an Androgen Excess Society guideline. J. Clin. Endocrinol. Metab. 91(11), 4237-4245 (2006)

54. E. Kousta et al., The prevalence of polycystic ovaries in women with infertility. Hum. Reprod. 14(11), 2720-2723 (1999)

55. A. Benrick et al., Resveratrol is not as effective as physical exercise for improving reproductive and metabolic functions in rats with dihydrotestosterone-induced polycystic ovary syndrome. Evid Based Complement Alternat Med, 2013. 2013: p. 964070

56. A. Mansour et al., Effect of resveratrol on menstrual cyclicity, hyperandrogenism and metabolic profile in women with PCOS. Clin. Nutr. 40(6), 4106-4112 (2021)

57. E. Papaleo et al., Myo-inositol in patients with polycystic ovary syndrome: a novel method for ovulation induction. Gynecol. Endocrinol. 23(12), 700-703 (2007)

58. A. Bevilacqua et al., Results from the International Consensus Conference on myo-inositol and D-chiroinositol in Obstetrics and Gynecology-assisted reproduction technology. Gynecol Endocrinol, 2015. 31(6): p. 441-6

59. R.S. Legro et al., Prevalence and predictors of risk for type 2 diabetes mellitus and impaired glucose tolerance in polycystic ovary syndrome: a prospective, controlled study in 254 affected women. J. Clin. Endocrinol. Metab. 84(1), 165-169 (1999)

60. R.M. Ortega, C. Pérez-Rodrigo, A.M. López-Sobaler, Dietary assessment methods: dietary records. Nutr. Hosp. 31(Suppl 3), 38-45 (2015)

61. A. Hoseini et al., The effects of resveratrol on metabolic status in patients with type 2 diabetes mellitus and coronary heart disease. 2019. 10(9): p. 6042-6051

62. J.P. Crandall et al., Pilot study of resveratrol in older adults with impaired glucose tolerance. J. Gerontol. A Biol. Sci. Med. Sci. 67(12), 1307-1312 (2012)

63. S. Chen et al., Resveratrol improves insulin resistance, glucose and lipid metabolism in patients with non-alcoholic fatty liver disease: a randomized controlled trial. Dig. Liver Dis. 47(3), 226-232 (2015) 
64. E.O. Talbott et al., Polycystic ovarian syndrome (PCOS): a significant contributor to the overall burden of type 2 diabetes in women. J. Womens Health (Larchmt) 16(2), 191-197 (2007)

65. D. Costantino et al., Metabolic and hormonal effects of myo-inositol in women with polycystic ovary syndrome: A double-blind trial. Eur. Rev. Med. Pharmacol. Sci. 13, 105-110 (2009)

66. F. Fruzzetti et al., Comparison of two insulin sensitizers, metformin and myo-inositol, in women with polycystic ovary syndrome (PCOS). Gynecol. Endocrinol. 33(1), 39-42 (2017)

67. L.G. Cooney et al., High prevalence of moderate and severe depressive and anxiety symptoms in polycystic ovary syndrome: a systematic review and meta-analysis. Hum. Reprod. 32(5), 1075-1091 (2017)

68. H.J. Teede et al., Recommendations from the international evidence-based guideline for the assessment and management of polycystic ovary syndromełł. Hum. Reprod. 33(9), 1602-1618 (2018)

69. A. Dokras, Mood and anxiety disorders in women with PCOS. Steroids 77(4), 338-341 (2012)

70. J.A. Barry, A.R. Kuczmierczyk, P.J. Hardiman, Anxiety and depression in polycystic ovary syndrome: a systematic review and meta-analysis. Hum. Reprod. 26(9), 2442-2451 (2011)

71. A.A. Deeks et al., Is having polycystic ovary syndrome a predictor of poor psychological function including anxiety and depression? Hum. Reprod. 26(6), 1399-1407 (2011)

72. T. Liu et al., Resveratrol ameliorates estrogen deficiency-induced depression- and anxiety-like behaviors and hippocampal inflammation in mice. Psychopharmacol. (Berl) 236(4), 1385-1399 (2019)

73. M.H. Farzaei et al., Effect of resveratrol on cognitive and memory performance and mood: A metaanalysis of 225 patients. Pharmacol. Res. 128, 338-344 (2018)

74. A.Y. Berman et al., The therapeutic potential of resveratrol: a review of clinical trials. NPJ Precis Oncol, 2017. 1

\section{Tables}

Table.1. Paired sample t-test comparing the anthropometric, endocrine and metabolic parameters in PCOS before and after three months of treatment with Metformin + Pioglitazone and Resveratrol + Myoinositol. 


\begin{tabular}{|c|c|c|c|c|c|c|}
\hline & Metformin + Pios & itazone & & Resveratrol + & Myoinositol & \\
\hline Variable & Afte & 12 weeks & & seline & After 12 week & \\
\hline D & $(n=55)$ & $(n=44)$ & $\mathbf{P}$ & $(n=55)$ & $(n=44$ & \\
\hline Anthropometric features & & & & & & \\
\hline Weight (Kgs) & $\begin{array}{l}80.07 \pm \\
11.329\end{array}$ & $\begin{array}{l}80.51 \pm \\
10.746\end{array}$ & .264 & $\begin{array}{l}85.75 \pm \\
13.650\end{array}$ & $\begin{array}{l}80.32 \pm \\
12.232\end{array}$ & $<0.001$ \\
\hline BMI (kg/m2) & $\begin{array}{l}33.10 \pm \\
4.676\end{array}$ & $\begin{array}{l}33.20 \pm \\
4.385\end{array}$ & .575 & $\begin{array}{l}33.86 \pm \\
4.527\end{array}$ & $\begin{array}{l}31.48 \pm \\
4.100\end{array}$ & $<0.001$ \\
\hline SBP $(\mathrm{mmHg})$ & $\begin{array}{l}123.98 \pm \\
7.438\end{array}$ & $\begin{array}{l}122.80 \pm \\
5.424\end{array}$ & .247 & $\begin{array}{l}125.80 \pm \\
6.984\end{array}$ & $\begin{array}{l}121.89 \pm \\
5.772\end{array}$ & .002 \\
\hline $\mathrm{DBP}(\mathrm{mmHg})$ & $\begin{array}{l}79.66 \pm \\
6.138\end{array}$ & $\begin{array}{l}80.27 \pm \\
5.808\end{array}$ & .453 & $\begin{array}{l}82.16 \pm \\
7.021\end{array}$ & $\begin{array}{l}77.50 \pm \\
5.757\end{array}$ & .001 \\
\hline Pulse (mins) & $\begin{array}{l}72.25 \pm \\
2.721\end{array}$ & $\begin{array}{l}71.91 \pm \\
1.939\end{array}$ & .423 & $\begin{array}{l}74.45 \pm \\
4.151\end{array}$ & $\begin{array}{l}72.43 \pm \\
1.771\end{array}$ & .008 \\
\hline FG score & $\begin{array}{l}13.41 \pm \\
3.405\end{array}$ & $\begin{array}{l}12.34 \pm \\
3.660\end{array}$ & .010 & $\begin{array}{l}13.36 \pm \\
1.844\end{array}$ & $\begin{array}{l}10.32 \pm \\
1.667\end{array}$ & .008 \\
\hline Waist (cm) & $\begin{array}{l}104.89 \pm \\
11.479\end{array}$ & $\begin{array}{l}105.23 \pm \\
10.877\end{array}$ & .430 & $\begin{array}{l}105.95 \pm \\
12.588\end{array}$ & $\begin{array}{l}97.84 \pm \\
11.936\end{array}$ & $<0.001$ \\
\hline Hip (cm) & $\begin{array}{l}112.18 \pm \\
10.289\end{array}$ & $\begin{array}{l}112.64 \pm \\
10.070\end{array}$ & .265 & $\begin{array}{l}116.09 \pm \\
11.775\end{array}$ & $\begin{array}{l}111.98 \pm \\
11.143\end{array}$ & $<0.001$ \\
\hline WHR & $\begin{array}{l}.9295 \pm \\
.03976\end{array}$ & $\begin{array}{l}.9293 \pm . \\
05055\end{array}$ & .954 & $\begin{array}{l}.9070 \pm \\
.05219\end{array}$ & $\begin{array}{l}.8580 \pm \\
.05179\end{array}$ & $<0.001$ \\
\hline Hormonal profile & & & & & & \\
\hline FSH (mlU/ml) & $\begin{array}{l}6.580 \pm \\
3.7045\end{array}$ & $\begin{array}{l}7.179 \pm \\
3.9725\end{array}$ & .108 & $\begin{array}{l}8.165 \pm \\
4.2058\end{array}$ & $\begin{array}{l}6.116 \pm \\
2.3005\end{array}$ & $<0.001$ \\
\hline LH (mlU/ml) & $\begin{array}{l}9.875 \pm \\
6.3230\end{array}$ & $\begin{array}{l}8.848 \pm \\
3.3331\end{array}$ & .209 & $\begin{array}{l}14.753 \pm \\
6.0492\end{array}$ & $\begin{array}{l}9.045 \pm \\
3.2399\end{array}$ & $<0.001$ \\
\hline Testotosterone* $(\mathrm{ng} / \mathrm{ml})$ & $\begin{array}{l}1.00(0.73- \\
1.38)\end{array}$ & $\begin{array}{l}1.22(0.80- \\
1.85)\end{array}$ & .193 & $\begin{array}{l}0.65(0.55- \\
0.77)\end{array}$ & $\begin{array}{l}0.37(0.27- \\
0.51)\end{array}$ & $<0.001$ \\
\hline Metabolic parameter & & & & & & \\
\hline Insulin* $(\mu \mathrm{IU} / \mathrm{ml})$ & $\begin{array}{l}21.53(16.95- \\
27.35)\end{array}$ & $\begin{array}{l}23.17(18.55- \\
28.94)\end{array}$ & .565 & $\begin{array}{l}40.43(30.4- \\
53.6)\end{array}$ & $\begin{array}{l}25.57(18.9- \\
34.5)\end{array}$ & .002 \\
\hline
\end{tabular}


Data for the continuous variables is presented as mean \pm standard deviation. P value shows the difference between the two groups before and after treatment. BMI; body mass index, SBP\&DBP; Systolic and diastolic blood pressure, WHR; waist hip ratio, $L H$; luteinizing hormone, $F S H$; follicle stimulating hormone, PSS; perceived stress scores, *log transformed for analysis, values presented as geometric mean (confidence interval)

Table.2. Chi-Square test comparing basal characteristics of the participants included in the study and their response to the treatment regimens in the two treatment arms based on menstrual irregularity and acne.

$\begin{array}{rlll}\text { Metformin }+ & \text { Resveratrol }+ & & \text { Chi-S } \\ \text { Pioglitazone } & \text { Myoinositol } & \\ \mathrm{n}= & \begin{array}{l}\mathrm{n}=\mathrm{m} \text { within } \\ \text { within }\end{array} & \text { Total } & \mathrm{p}= \\ \text { group } & & & \end{array}$

group

Menstrual Irregularity B

Regular

Irregular

$44 \quad 100 \%$

$44 \quad 100 \%$

$88 \quad 100 \%$

Menstrual Irregularity After

\begin{tabular}{llllllll} 
Regular & 11 & $18.2 \%$ & 37 & $84.1 \%$ & 48 & $51.1 \%$ & 0.001 \\
\cline { 1 - 5 } Irregular & 36 & $81.8 \%$ & 7 & $15.9 \%$ & 43 & $48.9 \%$ & \\
\cline { 1 - 5 }
\end{tabular}

Acne Before

\begin{tabular}{llllllll} 
No & 20 & $38.6 \%$ & 30 & $68.2 \%$ & 50 & $53.4 \%$ & 0.10 \\
\cline { 1 - 5 } Yes & 27 & $61.4 \%$ & 14 & $31.8 \%$ & 41 & $46.6 \%$ &
\end{tabular}

Acne After

\begin{tabular}{llllllll}
\cline { 1 - 3 } No & 22 & $43.2 \%$ & 40 & $90.9 \%$ & 62 & $67 \%$ & $<0.001$ \\
\cline { 1 - 6 } Yes & 25 & $56.8 \%$ & 4 & $9.1 \%$ & 29 & $33 \%$ &
\end{tabular}

\section{Figures}




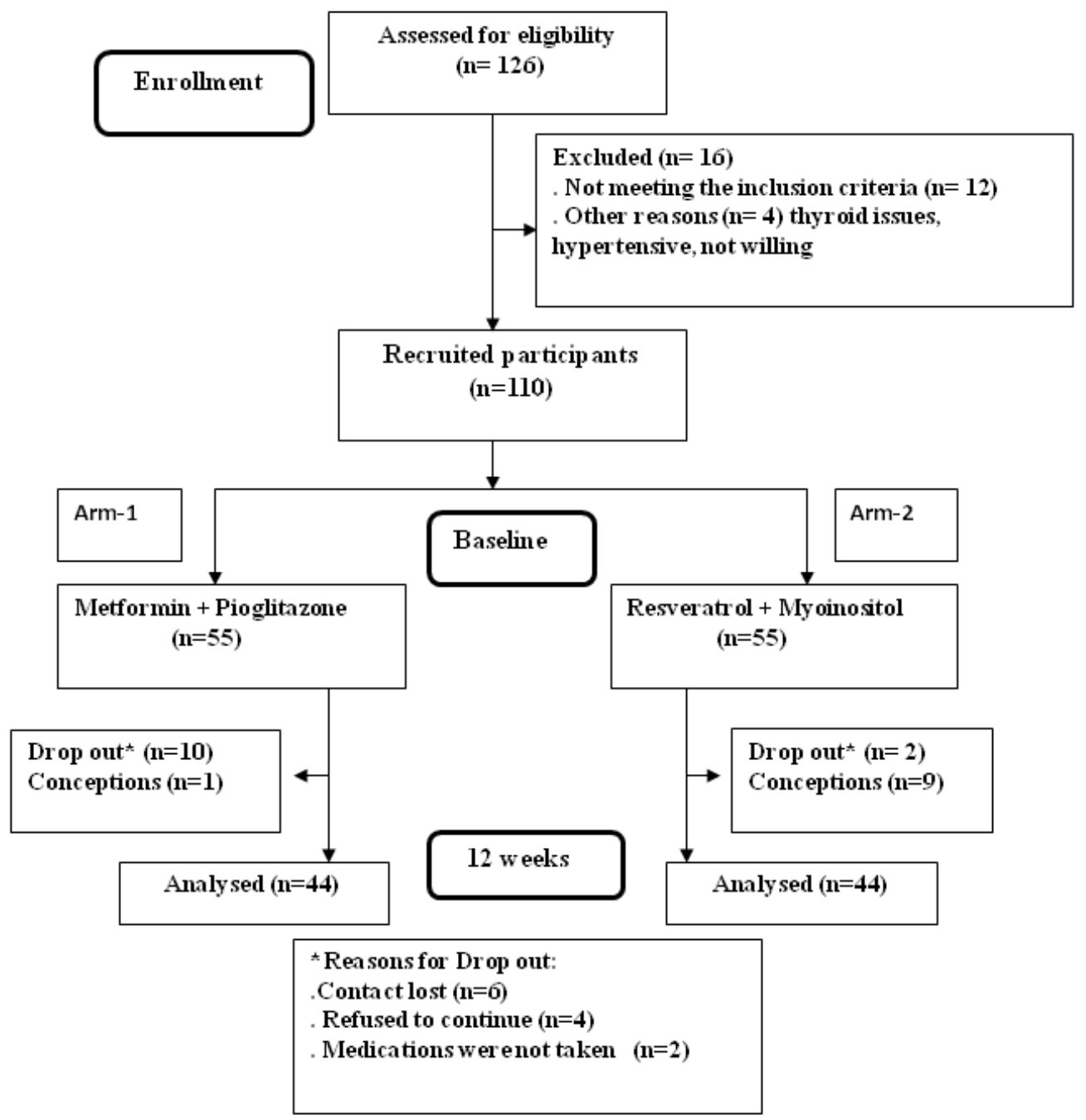

Figure 1

Shows the flow diagram of the trial from recruitment to the end of the 3 months of treatment with combined metformin + Pioglitazone and Resveratrol + Myoinositol 


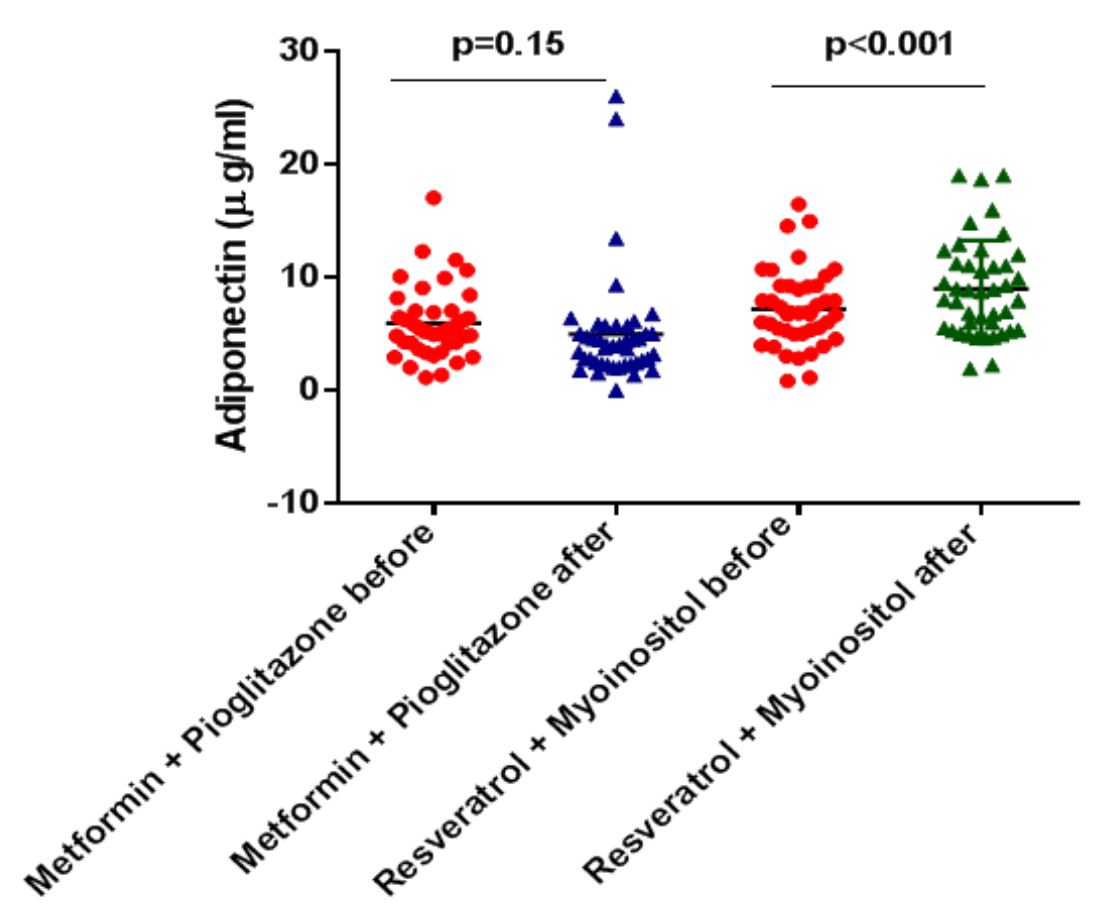

Figure 2

PCOS decrease adiponectin level and treatment with combined resveratrol and myoinositol increases adiponectin level in women with PCOS. A. Experimental design: Red circle represents baseline value; Blue triangle represents conventional arm-1, Metformin + Pioglitazone; green triangle represents interventional arm-2, Resveratrol + Myoinositol; triangles facing upward \& downward represent increase and decrease respectively B. Prior to the treatment adiponectin levels are low in both the study arms. Treatment with resveratrol and myoinositol noteworthy increased the serum adiponectin level $(p=<0.001)$. No significant improvement was seen in Metformin and Pio arm ( $p=0.15)$ 


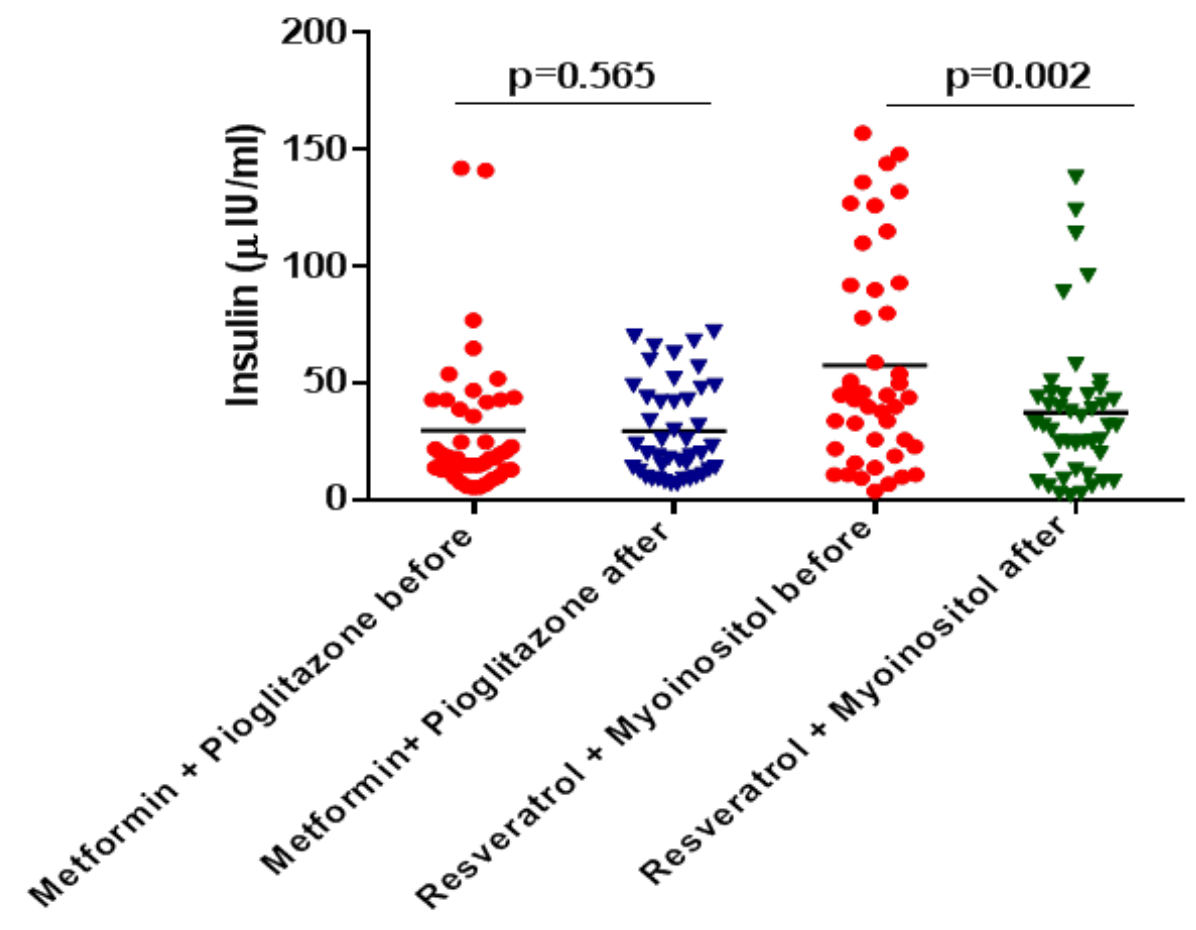

\section{Figure 3}

Serum insulin level is increased due to insulin resistance in PCOS affected women. The baseline serum insulin level was high in both the arms. A significant diminution in the level of serum insulin was observed in the Resveratrol and myoinositol treated women $(p=0.002)$. No significant reduction was observed with the metformin and pioglitazone treatment $(p=0.565)$. 
A

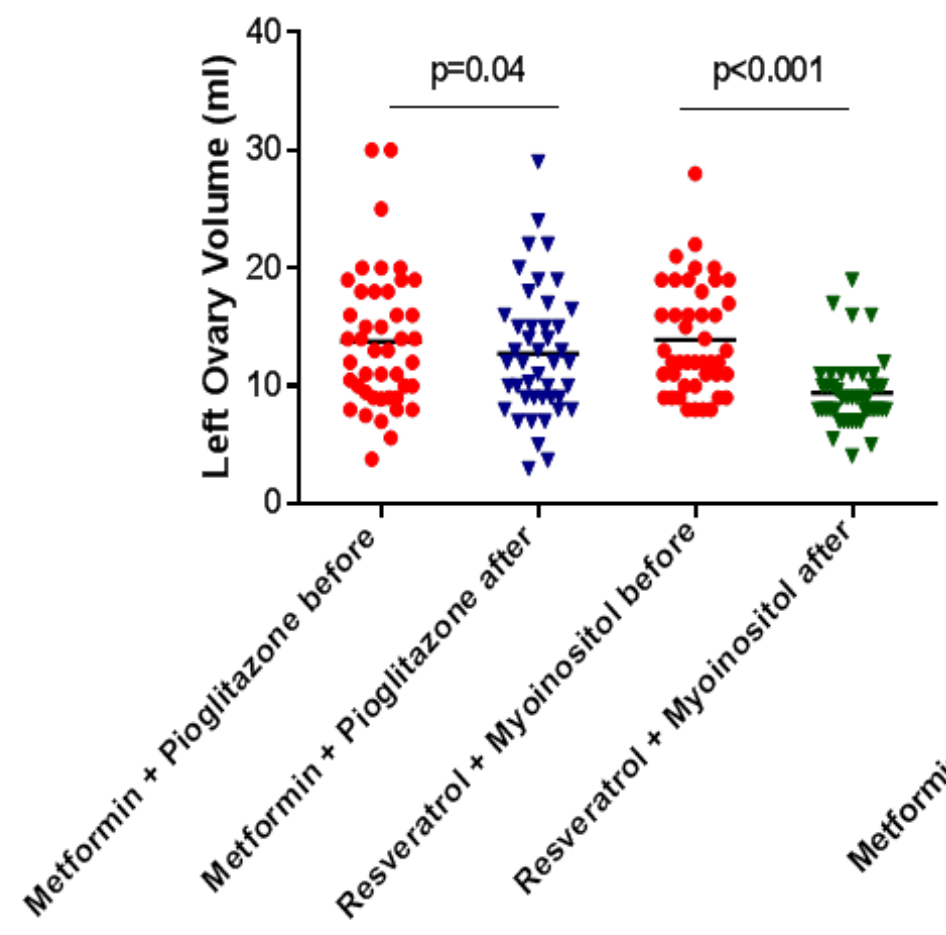

B

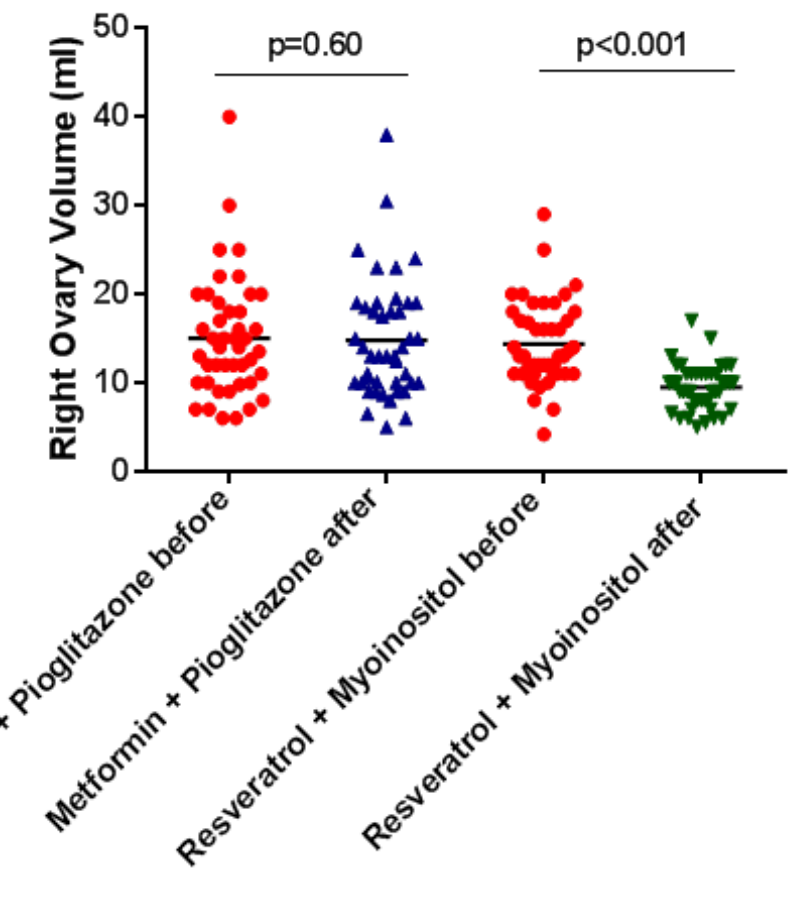

\section{Figure 4}

Ovarian volume in PCOS is increased due to multiple cysts. A. The baseline right sided ovarian volume was extremely high in both the treatment arms. Treatment after 12 weeks with resveratrol and myoinositol significantly reduced the ovarian volume in the right ovary $(p<0.001)$. The reduction was not significant in the metformin and Pioglitazone treated participants $(p=0.60)$; B. the baseline, left sided ovarian volume was extremely high in participants of both the treatment arms. Treatment after 12 weeks reduced the volume in both the arms but the reduction in the left ovary was highly significant in the resveratrol and myoinositol arm $(p<0.001)$ when compared to the metformin and pioglitazone arm $(p=0.04)$. 


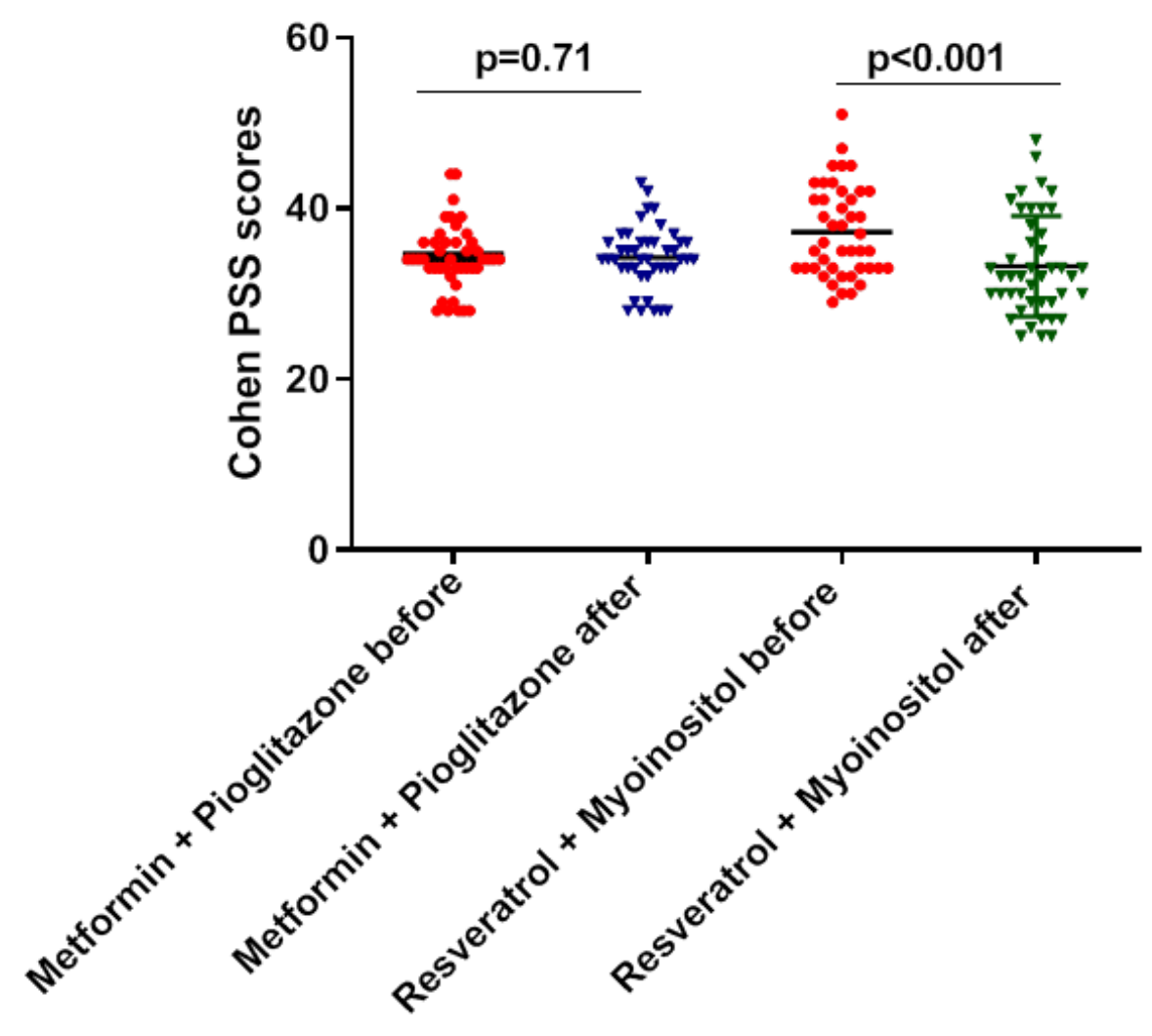

\section{Figure 5}

Shows the stress scores at baseline and after 12 weeks treatment in the Metformin + Pioglitazone and resveratrol + Myoinositol arm. Post treatment shows the significant alleviation in the Resveratrol + Myoinositol arm $(p<0.001)$. No significant reduction in Metformin + Pioglitazone arm $(p=0.71)$. 